\title{
Impact of the diagnostic changes to post-traumatic stress disorder for DSM-5 and the proposed changes to ICD-11
}

\author{
Meaghan L. O'Donnell, Nathan Alkemade, Angela Nickerson, Mark Creamer, \\ Alexander C. McFarlane, Derrick Silove, Richard A. Bryant and David Forbes
}

\section{Background}

There have been changes to the criteria for diagnosing post-traumatic stress disorder (PTSD) in DSM-5 and changes are proposed for ICD-11.

\section{Aims}

To investigate the impact of the changes to diagnostic criteria for PTSD in DSM-5 and the proposed changes in ICD-11 using a large multisite trauma-exposed sample and structured clinical interviews.

\section{Method}

Randomly selected injury patients admitted to four hospitals were assessed 72 months post trauma $(n=510)$. Structured clinical interviews for PTSD and major depressive episode, as well as self-report measures of disability and quality of life were administered.

\section{Results}

Current prevalence of PTSD under DSM-5 scoring was not significantly different from DSM-IV (6.7\% v. 5.9\%, $z=0.53$, $P=0.59$ ). However, the ICD-11 prevalence was significantly lower than ICD-10 (3.3\% v. 9.0\%, $Z=-3.8, P<0.001)$. The
PTSD current prevalence was significantly higher for DSM-5 than ICD-11 (6.7\% v. 3.3\%, $Z=2.5, P=0.01)$. Using ICD-11 tended to show lower rates of comorbidity with depression and a slightly lower association with disability.

\section{Conclusions}

The diagnostic systems performed in different ways in terms of current prevalence rates and levels of comorbidity with depression, but on other broad key indicators they were relatively similar. There was overlap between those with PTSD diagnosed by ICD-11 and DSM-5 but a substantial portion met one but not the other set of criteria. This represents a challenge for research because the phenotype that is studied may be markedly different according to the diagnostic system used.

\section{Declaration of interest}

R.A.B. served on the DSM-5 PTSD/Trauma/Dissociative Work Group. These comments reflect the opinions of the author and not necessarily those of the American Psychiatric Association or of the DSM-5 Work Groups.
The next few years represent a critical time for the field of traumatic stress. There have been changes to the diagnostic criteria for post-traumatic stress disorder (PTSD) in the DSM- $5^{1}$ and other changes are proposed for ICD-11 (http://apps.who.int/ classifications/icd11/browse/l-m/en). The approach taken by these classification schemes to the diagnosis of PTSD is markedly different. Released in May 2013, DSM-5 adopted a clinical approach, expanding the symptom criteria to capture a more comprehensive profile of the post-traumatic stress response ${ }^{2}$ including symptoms that may be shared with other disorders. To mitigate against the potential for higher rates of comorbidity resulting from shared symptomology, the number of key symptom clusters in DSM-5 has been increased from three to four. In contrast, the ICD-11 committee has adopted a more focused approach that recognises the central role of re-experiencing fear. ${ }^{3,4}$ In this regard, the direction being pursued for ICD-11 is to identify the symptoms unique to the PTSD construct, ${ }^{4}$ avoiding symptoms that overlap with other disorders such as depression. To date, few studies have examined the impact of these changes on prevalence rates of PTSD.

The main criteria changes for DSM-5, compared with DSM-IV, ${ }^{5}$ include the removal of Criterion A2 (subjective response to the traumatic event), the separating of active avoidance from passive avoidance (Criterion $\mathrm{C}$ ), and the creation of a new cluster of symptoms (Criterion D - Negative alterations in cognitions and mood). The hyperarousal cluster (Criterion D in DSM-IV) generally remains the same but is now Criterion E. In ICD-11 the definition of a traumatic event places greater emphasis on clinical judgement, compared with ICD-10, ${ }^{6}$ and is no longer described as one that likely causes pervasive distress in almost anyone. Re-experiencing symptoms are contained to those research findings unique to PTSD, namely flashback and nightmares. Like DSM-5, ICD-11 has focused on active avoidance, rather than passive. The number of hyperarousal symptoms is reduced to two: hypervigilance and exaggerated startle. At least one re-experiencing, one avoidance and one hyperarousal symptom is required. A duration and functional impairment criteria are now included. The purpose of this study is to explore how these different approaches affect rates of current PTSD, the impact on the relationship with depression comorbidity, and the relationship with disability and quality of life.

\section{Method}

\section{Participants}

These data were collected as part of a larger study of the mental health impact of severe injury, the Australian Vulnerability study. Injury patients were recruited from four hospitals in three states of Australia (in the period April 2004 to February 2006). All patients were admitted into the trauma service for at least $24 \mathrm{~h}$ and met the DSM-IV Criterion A1. ${ }^{5}$ Approval for this study was gained from the Human Research Ethics Committee (HREC) at each hospital. Patients were recruited to the study if they had experienced a serious injury that required a hospital admission of greater than $24 \mathrm{~h}$; were aged between 16 and 70 years; and had sufficient English comprehension to complete the assessment. Patients were included if they experienced a mild traumatic brain injury ${ }^{7}$ but were excluded if the experienced traumatic brain injury was more 
severe, or they were currently suicidal or psychotic. A mild traumatic brain injury was defined using the American Congress of Rehabilitation Medicine definition as a loss of consciousness of approximately $30 \mathrm{~min}$ or less or a Glasgow Coma Scale score of 13-15 after 30 min or post-traumatic amnesia not greater than $24 \mathrm{~h}^{7}$

Weekday trauma service admissions were randomly selected into the study over a 22-month period. Only weekday admissions were included because of limitations in recruitment resources. Random selection occurred through an automated procedure, stratified by length of stay. Random selection was used because the numbers of patients admitted to each trauma service was far greater than the study's recruitment resources allowed. Of the 1590 patients eligible for the study, 953 participants consented to participation and completed the baseline questionnaires for this study. At 72 months, 510 (54\% of initial participants) completed the assessment, which represented $32 \%$ of all eligible patients.

Individuals who refused to participate in the study did not differ from participants in gender, the presence of a mild traumatic brain injury, education, mechanism of injury, length of stay or Injury Severity Score (ISS). ${ }^{8}$ Those who did not complete the 72-month assessment did not differ from those who were recruited in terms of gender, the presence of a mild traumatic brain injury, education, mechanism of injury, length of stay or ISS. Those who did not complete the 72-month assessment differed from completers in that they were more likely to be younger (mean $36.48 \quad($ s.d. $=13.80)$ v. mean 39.52 (s.d.=13.35), $t(1108)=-3.72, \quad P<0.001)$ and have higher baseline Clinician-Administered PTSD Scale for DSM-IV (CAPS) ${ }^{9}$ scores (mean 19.67 (s.d.=17.62) v. mean 16.60 (s.d.=15.42), $t(1100)=3.05, P=0.002)$.

Of those who completed the 72-month assessment, the majority were male $(71 \%, n=362)$ which is typical of an Australian injury sample. ${ }^{10}$ Half the sample was married or in a relationship equivalent to a common law marriage (53\%, $n=245$ ). On average patients spent 12.35 (s.d. $=12.83$ ) days in hospital. The mean ISS was 10.6 (s.d.=7.26), which is in the moderate severity range. A total of $41 \%$ of participants $(n=209)$ experienced a mild traumatic brain injury. ${ }^{7}$ The principal mechanism of injury was a transport accident $(66 \%, n=335)$, followed by falls $(17 \%, n=86)$, assault $(6 \%, n=30)$, work-related accidents not specified in the above categories $(5 \%, n=26)$ and other $(7 \%, n=33)$.

\section{Measures}

PTSD

Symptom severity and diagnosis of PTSD were assessed using the CAPS. ${ }^{9}$ This structured clinical interview is one of the most widely used tools for diagnosing PTSD and has demonstrated excellent reliability and validity. ${ }^{11}$ The new questions proposed for DSM-5 were written by the CAPS original authors and incorporated into the interview. In the current study the CAPS internal consistency was high in both the DSM-IV $(\alpha=0.88)$ and the DSM-5 $(\alpha=0.89)$ versions. The CAPS interviews were conducted via telephone, which have been shown to be as valid and reliable as face-to-face interviews. ${ }^{12}$ All interviews were digitally recorded to ensure ongoing adherence to the protocol. To test interrater reliability, 5\% of all CAPS interviews were assessed by an independent assessor who was masked to the original scoring. Overall, the diagnostic consistency on the CAPS was $100 \%$.

\section{Depression}

The Mini International Neuropsychiatric Interview Version 5.5 $(\mathrm{MINI})^{13}$ was used to assess a diagnosis of major depressive episode. The MINI is a short, structured screening interview based on DSM-IV and ICD-10 classification of mental illness. It consists of a set of screening questions and modules; modules are administered if a patient responds positively to the screening question. The major depressive episode module assesses all major depressive episode symptoms except the distress and impairment symptoms. The MINI has good reliability for all diagnoses when compared with the Composite International Diagnostic Interview (CIDI). ${ }^{13}$

\section{Functional outcomes}

We used disability and quality of life to identify functional and well-being outcomes associated with a PTSD diagnosis. Disability provides an understanding of the problems an individual is having in performing activities or roles within the context of his or her environment ${ }^{14}$ and quality of life provides information on how a disability may impact on broader aspects of well-being. ${ }^{15}$ We used the 12-item World Health Organization Disability Assessment Schedule II (WHODAS $\mathrm{II}^{15}$ ) to measure disability. The WHODAS II measures activity limitations across six domains: (a) understanding and communication, (b) getting around, (c) self-care, (d) getting along with others, (e) household and work activities, and (f) participation in society. Items are rated on a five-point scale. The WHODAS II has been shown to be a reliable and valid measure of disability across various patient groups. ${ }^{16}$ The method of scoring we used was a summing of all the items. ${ }^{17}$ Scores range from 0 (no disability) to 48 (complete disability). There is no agreed cut-point for identifying people with significant disability, but people scoring 10-48 are in the top $10 \%$ of the population distribution of WHODAS II scores and are likely to have clinically significant disability. ${ }^{17}$ Thus, we used a score $>9$ as the threshold for high disability.

We used the psychological domain scale from the World Health Organization Quality of Life - BREF (WHOQOL-BREF ${ }^{18}$ ) as a measure of quality of life. The WHOQOL-BREF psychological domain is an eight-item scale that assesses quality of life in terms of perception and satisfaction across a number of life areas. The WHOQOL-BREF demonstrates good discriminant validity, content validity, internal consistency and test-retest reliability. ${ }^{18}$ In the current study, a scoring algorithm was used to standardise scores to a $0-100$ scale $^{15}$ with higher scores indicating higher quality of life. Australian population norms were used to identify thresholds and a score of less than 55.5 was used as the cut-off for poor psychological quality of life. ${ }^{19}$

\section{Procedure}

The study was fully explained to the patients who met inclusion criteria and written informed consent was obtained. Demographic and injury information was collected at baseline (just prior to discharge). We administered the CAPS at baseline and these data were used for completer analyses. At the 72-month follow-up, both the CAPS and MINI were administered via the telephone. Self-report questionnaires were sent to participants to assess disability and quality of life. The major analyses in this manuscript utilise the 72-month data.

\section{Data analysis}

Descriptive statistics were used to report the current prevalence rates associated with each scoring algorithm. To test the predictive power of each scoring algorithm, we examined the relationship between the diagnosis and poor psychological quality of life and high disability. We used measures of sensitivity to examine the probability that an individual with a poor psychological quality of life (or high disability) would have met criteria for a diagnosis; 
specificity to examine the probability that an individual without a poor psychological quality of life (or high disability) did not meet criteria for a diagnosis; positive predictive power to examine the probability that an individual who met the diagnostic criteria also met criteria for low psychological quality of life (or high disability); and negative predictive power to examine the probability that an individual without the diagnosis did not meet criteria for low psychological quality of life (or high disability).

\section{Results}

\section{Current prevalence}

The current prevalence rates of PTSD, as scored using DSM-IV, DSM-5, ICD-10 and ICD-11 algorithms are reported in Table 1. Rates of PTSD as scored for DSM-5 were higher than DSM-IV, however, this increase was not significant $(6.7 \%$ v. $5.9 \%$, $z=0.53, \quad P=0.59$ ). Most of this difference, however, was accounted for by the inclusion of Criterion A2 in DSM-IV. When A2 was removed as a requirement for the DSM-IV diagnosis, the rates of PTSD were higher for DSM-IV than DSM-5 (8.0\% $v$. $6.7 \%)$, although this difference was not significant $(z=0.80$, $P=0.42$ ). Aside from the A2 issue, of those who met DSM-IV but not DSM-5 criteria, 63\% were excluded from the latter diagnostic system because they did not meet the new requirement for active avoidance symptoms.

Rates of PTSD as scored using the proposed ICD-11 criteria were significantly lower than for the ICD-10 criteria $(3.3 \% v$. 9.0\%; $z=-3.8, P<0.001)$. The individuals diagnosed with PTSD using ICD-10 criteria that did not make an ICD-11 diagnosis failed to meet the re-experiencing symptom requirements $(30 \%$ of those with a diagnosis using ICD-10, $n=9$ ), the arousal requirements $(30 \%, n=9)$ and the functional impairment requirement $(13 \%, n=4)$. If intrusive memories was added as a re-experiencing symptom (in addition to flashbacks and nightmares), the current prevalence of PTSD scored by ICD-11 increased to $6.1(n=31)$.

Post-traumatic stress disorder current prevalence rates were significantly higher for DSM-5 compared with ICD-11 criteria (6.7\% v. 3.3\%, $z=2.5, P=0.01)$. Fifteen participants (42\%) met both DSM-5 and ICD-11 criteria, whereas 2 met ICD-11 but not DSM-5 and 19 met DSM-5 but not ICD-11. When DSM-IV was compared with DSM-5, 22 participants met criteria for both, 12 for DSM-5 only and 8 for DSM-IV only. Comparing ICD-10 with ICD-11 indicated that 30 participants met criteria for both, 16 for ICD-10 only and 1 for ICD-11 only. Table 2 breaks this down further by showing the proportion of the sample meeting diagnostic criteria for each cluster of symptoms across diagnostic algorithms.

\section{Comorbidity}

Comorbidity with depression was similar using DSM-IV and DSM-5 (69\% v. 67\%, $z=0.17, P=0.87)$. There was no difference in the proportion of participants diagnosed with PTSD using ICD-10 and ICD-11 with comorbid depression (56\% for ICD-10 v. $56 \%$ for ICD-11). Although not significantly different, participants diagnosed with PTSD using DSM-5 had 11\% higher comorbidity with depression compared with those diagnosed using ICD-11 (67\% v. $56 \%, z=-0.75, P=0.45)$.

\section{Functional outcome}

There was little difference in the proportion of participants diagnosed with PTSD using DSM-IV or DSM-5 that met criteria for high disability (92\% in DSM-IV v. $86 \%$ in DSM-5, $z=0.70$, $P=0.48)$ or poor psychological quality of life $(81 \%$ v. $75 \%$; $z=0.53, P=0.60)$. Similarly, there were no differences in the proportion of participants diagnosed with PTSD using ICD-10 or ICD-11 that met criteria for high disability $(85 \% v .77 \% ; z=0.67$, $P=0.50)$ or quality of life $(65 \% v .69 \% ; z=-0.26, P=0.79)$.

Table 1 Post-traumatic stress disorder (PTSD) 'caseness', comorbidity with depression and disability caseness as scored by different PTSD algorithms $(n=510)$

\begin{tabular}{|lcccc|}
\cline { 2 - 5 } & $\begin{array}{c}\text { PTSD caseness } \\
(n=510)\end{array}$ & $\begin{array}{c}\text { Proportion of participants with }(n / N) \\
\text { PTSD comorbid with a depression } \\
\text { diagnosis }^{\mathrm{a}}(n=507)\end{array}$ & $\begin{array}{c}\text { Proportion of participants } \\
\text { with PTSD meeting disability } \\
\text { caseness }^{\mathrm{b}}(n=450)\end{array}$ & $\begin{array}{c}\text { Proportion of participants with PTSD } \\
\text { meeting poor psychological quality } \\
\text { of life caseness }^{\mathrm{c}}(n=452)\end{array}$ \\
\hline DSM-IV with A2 & $5.9(30)$ & $69(20 / 29)$ & $92(24 / 26)$ & $81(21 / 26)$ \\
\hline DSM-IV without A2 & $8.0(41)$ & $63(25 / 40)$ & $83(30 / 36)$ & $72(26 / 36)$ \\
\hline DSM-5 & $6.7(34)$ & $67(22 / 33)$ & $85(24 / 28)$ & $75(21 / 28)$ \\
\hline ICD-10 & $9.0(46)$ & $56(25 / 45)$ & $77(10 / 13)$ & $65(26 / 40)$ \\
\hline ICD-11 & $3.3(17)$ & & $69(9 / 13)$ \\
\hline $\begin{array}{l}\text { a. Using the Mini International Neuropsychiatric Interview Version. } \\
\text { b. Using the World Health Organization Disability Assessment Schedule II. } \\
\text { c. Using the World Health Organization Quality of Life - BREF. }\end{array}$
\end{tabular}

Table 2 Endorsement of each set of criteria as defined by DSM-IV, DSM-5, ICD-10 and ICD-11 ( $n=510)$

\begin{tabular}{|c|c|c|c|c|}
\hline & \multicolumn{4}{|c|}{$\%(n)$} \\
\hline & DSM-IV & DSM-5 & ICD-10 & ICD-11 \\
\hline Criterion $\mathrm{A}^{\mathrm{a}}$ & $406(80)$ & $510(100)$ & $510(100)$ & $510(100)$ \\
\hline Re-experiencing & $112(22)$ & $74(15)$ & 97 (19) & $47(9)$ \\
\hline Avoidance & $86(17)$ & 74 (15) & $74(15)$ & $74(15)$ \\
\hline Mood disturbance & n/a & 96 (19) & $\mathrm{n} / \mathrm{a}$ & $\mathrm{n} / \mathrm{a}$ \\
\hline Arousal & $138(27)$ & $140(28)$ & $221(43)$ & $89(18)$ \\
\hline Impairment & $158(31)$ & $158(31)$ & $\mathrm{n} / \mathrm{a}$ & $158(31)$ \\
\hline
\end{tabular}




\begin{tabular}{|c|c|c|c|c|c|}
\hline & $\begin{array}{l}\text { Sensitivity } \\
(95 \% \mathrm{Cl})\end{array}$ & $\begin{array}{l}\text { Specificity } \\
(95 \% \mathrm{Cl})\end{array}$ & $\begin{array}{l}\text { Positive predictive } \\
\text { power }(95 \% \mathrm{Cl})\end{array}$ & $\begin{array}{l}\text { Negative predictive } \\
\text { power }(95 \% \mathrm{Cl})\end{array}$ & $\begin{array}{c}\text { Overall diagnostic } \\
\text { power }\end{array}$ \\
\hline \multicolumn{6}{|l|}{ Disability } \\
\hline DSM-IV & $0.16(0.11-0.24)$ & $0.99(0.97-1.00)$ & $0.92(0.73-0.99)$ & $0.71(0.66-0.75)$ & 0.72 \\
\hline DSM-5 & $0.16(0.11-0.23)$ & $0.99(0.96-1.00)$ & $0.86(0.66-0.95)$ & $0.71(0.66-0.75)$ & 0.72 \\
\hline ICD-10 & $0.23(0.17-0.31)$ & $0.98(0.96-0.99)$ & $0.85(0.69-0.94)$ & $0.72(0.68-0.77)$ & 0.74 \\
\hline ICD-11 & $0.07(0.03-0.12)$ & $0.99(0.97-1.00)$ & $0.77(0.46-0.94)$ & $0.69(0.64-0.73)$ & 0.69 \\
\hline \multicolumn{6}{|c|}{ Quality of life } \\
\hline DSM-IV & $0.17(0.11-0.26)$ & $0.98(0.96-0.99)$ & $0.81(0.60-0.93)$ & $0.77(0.72-0.80)$ & 0.77 \\
\hline DSM-5 & $0.17(0.11-0.26)$ & $0.98(0.96-0.99)$ & $0.75(0.55-0.89)$ & $0.76(0.72-0.80)$ & 0.76 \\
\hline ICD-10 & $0.21(0.15-0.30)$ & $0.96(0.93-0.98)$ & $0.65(0.48-0.79)$ & $0.77(0.73-0.81)$ & 0.76 \\
\hline ICD-11 & $0.07(0.04-0.14)$ & $0.99(0.97-1.00)$ & $0.69(0.39-0.90)$ & $0.75(0.70-0.78)$ & 0.74 \\
\hline
\end{tabular}

An important question, however, is whether one system is better than the other at identifying those who have functional impairment. Table 3 reports the sensitivity, specificity, positive predictive power and negative predictive power for the various diagnostic algorithms in relation to both disability and quality of life. Very little difference was apparent between DSM-5 and ICD-11 (or their older counterparts) on these parameters, except that ICD-11 had a very low sensitivity for determining low quality of life or high disability.

There were eight people who met PTSD criteria for DSM-IV but not DSM-5. By looking at the functional outcomes of these people relative to those who did meet DSM-5 criteria we could see whether DSM-5 was identifying those with higher functional impairment. Independent samples $t$-test were carried out for WHODAS II and WHOQOL-BREF measures, comparing those diagnosed in DSM-IV only with those diagnosed in DSM-5. With the WHODAS II there was not a significant difference for those diagnosed under DSM-IV only (mean $16.0 \quad($ s.d.=8.2)) and DSM-5 conditions (mean $16.6 \quad(s . d .=7.7), \quad t(34)=-0.19$, $P=0.85$ ). This was also the case for the WHOQOL-BREF (mean 52.6 (s.d.=17.4) v. mean $44.6($ s.d. $=13.2), t(34)=1.40, P=0.17)$.

Similar results were found for ICD. There were 28 people who meet PTSD criteria for ICD-10 but not ICD-11. Overall there was no difference in quality of life between those diagnosed under ICD-10 only, relative to those meeting ICD-11 criteria (WHODAS II - ICD-10 only: mean 15.9 (s.d.=6.9); ICD-11: mean 15.5 (s.d.=8.0), $\quad t(39)=0.146, \quad P=0.89)$. There was a significant difference between ICD-10 and ICD-11 in that those who met ICD-11 only had significantly lower psychological quality of life than those who met ICD-10 criteria only (WHOQOL-BREF ICD-10: mean 52.1 (s.d.=11.9); ICD-11: mean 43.0 (s.d.=16.8), $t(39)=2.005, P<0.05$, Cohen's $d=0.63)$.

\section{Discussion}

\section{Main findings}

In this study we examined the impact of the changes in DSM-5 and the proposed changes in ICD-11 on current prevalence rates of PTSD, on comorbidity rates with major depressive episode, and on the association between diagnosis, disability and quality of life. Overall, there were few differences between the DSM-IV and DSM-5 scoring algorithms in terms of current prevalence, comorbidity and their association with disability and quality of life. This was not the case with the ICD scoring algorithm, with the ICD11 having a significantly lower current prevalence rate than ICD-10.

\section{Comparision with findings from other studies}

The diagnostic requirements for PTSD in DSM-5 and the proposed criteria for ICD-11 have incorporated a number of modifications to their earlier counterpart classification systems. The only two studies to examine the prevalence rates of the DSM-5 criteria (which were under proposal at the time) provide conflicting results. The first study used a large sample of traumatic injury survivors $(n=835)$ but used only those criteria that are shared across both DSM-IV and DSM-5, focusing on the impact of splitting Cluster C to specifically require active avoidance. ${ }^{20}$ The prevalence of PTSD reduced by $26 \%$ as a result of this modification (80 individuals in DSM-IV-TR compared with 62 in DSM-5) and the prevalence of comorbidity between PTSD and major depression was reduced. The second study used a non-clinical university sample, ${ }^{21}$ raising questions about the extent to which the findings may generalise to the broader trauma-exposed community. Nevertheless, that study reported a small increase in prevalence with the revised criteria.

Much less detail and comment has appeared regarding the proposed changes to the ICD criteria for PTSD. It is 2 years behind the DSM revisions, with publication by the World Health Organization due in 2015. The key principles for the ICD-11 approach in general is an increased focus on the clinical utility of diagnoses and their accessibility to front-line workers. ${ }^{3,22}$ The emphasis on clinical utility encourages simplicity, which is important because ICD is applicable to the many low-income countries around the world with less developed mental health systems. The ICD-11 PTSD committee aimed to identify the symptoms specific to the disorder and separate these out from the non-specific components. ${ }^{3,22}$ The proposed specific criteria have only just been released for discussion ${ }^{3}$ and thus the criteria identified in this paper may change over time.

\section{Evaluation of our findings}

The decision to drop criterion A2 in DSM-5 was supported in our study. A total of $20 \%$ of those who would otherwise have met criteria for DSM-IV failed to get a diagnosis because they did not meet A2 criteria. Apart from the difference caused by A2, the current prevalence identified by DSM-5 compared with DSM-IV was lower and this was largely explained by some participants failing to meet the active avoidance cluster. This is consistent with the findings of Forbes et $a l^{20}$ and congruent with the assumption that active avoidance is a core part of this disorder.

If the goal of the ICD revision was to tighten the diagnosis, it seems to have succeeded: current prevalence dropped from $9.0 \%$ for ICD-10 to $3.3 \%$ for ICD-11. This was explained largely by the need to meet one of the limited number of re-experiencing and arousal symptoms in ICD-11, although the requirement for functional impairment also contributed to this drop. The constrained definition of re-experiencing in ICD-11, as a reliving of the event, emerges from models that emphasise this phenomenon as pivotal to $\mathrm{PTSD}^{23}$ and evidence that reliving is a feature that 
distinguished PTSD from other post-traumatic intrusive symptoms. ${ }^{24}$ Nevertheless, the scale of the reduction in the prevalence of PTSD raises questions as to whether the proposed criteria for ICD-11 may be too restrictive. Indeed, the current prevalence rate increased from $3.3 \%$ to $6.1 \%$ when distressing intrusive memories were included. It may be that the current operational definition of the re-experiencing symptoms requires further consideration to optimally capture this phenomenon of patients with PTSD.

One goal of modification for both systems was to improve the specificity of the diagnosis by increasing the emphasis on those symptoms that are unique to PTSD, such as active avoidance, and (for ICD at least) reducing those that represent general dysphoria or depression. If that goal were achieved, the revision would be expected to show a lower rate of comorbid depression. ${ }^{20}$ This was not supported by the findings for the DSM- 5 algorithm, which showed a comorbidity rate with depression of $67 \%$. A more detailed investigation of the relationship between DSM-5 PTSD symptoms clusters and depression is beyond the scope of this paper, but it would be a worthwhile endeavour for future investigation. Surprisingly, the ICD-11 algorithm also produced a high comorbidity with depression - half those with PTSD also met depression criteria. Given that this comorbidity could not be accounted for by overlapping symptoms, it would add weight to the view that PTSD and depression co-occur in the aftermath of trauma, independent of definitional overlap of diagnostic criteria. ${ }^{25}$ The chronicity of the current sample may contribute to this level of comorbidity, given that the index traumatic event occurred 6 years prior and there is evidence to suggest that depression and PTSD become indistinguishable as they become chronic. ${ }^{26}$

The final aim of this study was to explore the relationship of PTSD with functional outcomes under the different diagnostic algorithms. It is reasonable to assume that a disorder only reaches clinical significance when it impairs social or occupational functioning, or disrupts quality of life. Although ICD-11 showed particularly low sensitivity with high disability/low quality of life, there was surprisingly little difference across the various algorithms in terms of overall diagnostic accuracy (as seen by the overlapping confidence intervals). If therefore we wish to identify those whose mental health problems warrant intervention, there may be little to choose between the ICD and DSM revisions for PTSD. It is noted however, that those meeting ICD-11 only (relative to those meeting ICD-10 only) did have a significantly lower psychological quality of life, which adds support for this version of the criteria.

Since these diagnostic systems have taken quite different approaches - with DSM-5 taking an inclusive approach and ICD-11 tending towards a minimalist symptom list - it is not surprising that there were substantial differences between them in terms of the prevalence rates they generated, with the DSM-5 prevalence rate being significantly higher than ICD-11. However, what was more surprising was that the majority of individuals with PTSD were identified by one but not the other system - only $42 \%$ met the criteria of both systems. This difference is not explained by the finding that the DSM-5 diagnostic algorithm captured a larger group than the ICD-11, as only $12 \%$ of those with an ICD-11 diagnosis did not meet criteria for DSM-5. These findings indicate that the diagnostic algorithms of each system were predominantly identifying different people.

The apparent divergence in patients with PTSD identified by ICD-11 and/or DSM-5 is a key finding. There are both potential scientific and clinical consequences of having diagnostic systems that are not parallel. From a scientific perspective, attempts to understand the mechanisms underpinning PTSD may be hampered by diagnostic constructs that do not match replication and generalisation may be hindered by the lack of a standardised phenotype. Many initial findings about how PTSD works are not replicated, especially in relation to biological processes, and this has been attributed to the heterogeneity of the diagnostic definition; ${ }^{27}$ this situation may only be worsened by greater discrepancies between diagnostic systems. From a clinical perspective, how would compensation systems manage when a person may be entitled to compensation under one diagnostic system but not the other? Clinical interventions that may have been validated under one system may not be equally valid for the clinical manifestation of PTSD diagnosed under the alternate system. In short, it appears that the tendency for ICD-11 and DSM-5 to identify different trauma-affected people will promote less precision in the years ahead at both theoretical and applied levels.

\section{Strengths and limitations}

The current study had several strengths. The sample comprised a large multisite study of traumatised adults and the methodology involved using structured clinical interviews to diagnose PTSD and major depressive episode. Nevertheless, the limitations require consideration. First, given that sensitivity and positive predictive power are influenced by prevalence rates, ${ }^{28}$ the rate of PTSD in our sample would have contributed to the relatively low predictive power reported in our analyses. Similarly, a lack of power may have contributed to the non-significance of our $z$-score tests when assessing changes in prevalence or comorbidity rates using the different scoring algorithms. Second, since this sample represents those exposed to severe injury, other variables such as physical damage and pain may have contributed to the levels of dysphoria and functional impairment. Furthermore, the sample had a high rate of mild traumatic brain injury and it is unknown how this, or other injuries, may have had an impact on the prevalence rates of each symptom. Third, there was a non-participation bias towards higher baseline CAPS scores. This may have had an impact on the prevalence rates of PTSD, and/or the proportion of those meeting either ICD-11 or DSM-5 criteria. Finally, although the MINI is a well-validated structured screening interview, it is important to recognise that although it contains the nine depression symptoms it does not include the distress and impairment symptoms or the physiological exclusion criteria for major depressive episode, which may have had an impact on the prevalence rates of that disorder in the study.

\section{Implications}

Despite the limitations, the findings provide some cautious support for the DSM-5 revisions of the PTSD criteria and the proposed revisions to ICD-11. It was notable that the two systems resulted in significantly different prevalence rates, and that each identified a proportion of people with PTSD which the other system did not. This raises a challenge for future research because as previously highlighted the phenotype that is studied may be markedly different according to the diagnostic system used. This is especially important for international research given that many countries around the world employ the ICD, and research outcomes that use this system may be based on different study populations than those using DSM-based formula. It is important therefore that work aimed at refining and reconciling the diagnostic criteria for PTSD continues with the aim of achieving an empirically based unitary construct that accurately represents the disorder. 


\section{Funding}

This study was supported by a National Health and Medical Research Council Program Grant (568970).

\section{Acknowledgements}

The authors gratefully acknowledge all the participants involved in this study.

Meaghan L. O'Donnell, PhD, Nathan Alkemade, PhD, Australian Centre fo Posttraumatic Mental Health, Carlton, Victoria and Department of Psychiatry, University of Melbourne, Parkville, Victoria; Angela Nickerson, PhD, Schoo of Psychology University of New South Wales, Sydney, New South Wales; Mark Creamer, PhD, Department of Psychiatry, University of Melbourne, Parkville, Victoria; Alexander C. McFarlane, AO, MD, FRANZCP, Centre for Traumatic Stress, University of Adelaide, Adelaide, South Australia; Derrick Silove, MD, FRANZCP. University of Adelaide, Adelaide, South Australia; Derrick Silove, MD, FRANZCP,
Department of Psychiatry University of New South Wales, sydney and Mental Health Centre, Psychiatry Research and Teaching Unit, Liverpool, New South Wales; Richard A. Bryant, PhD, School of Psychology, University of New South Wales, Sydney, New South Wales; David Forbes, PhD, Australian Centre for Posttraumatic Mental Health, Carlton, Victoria and Department of Psychiatry, University of Melbourne, Parkville, Victoria, Australia

Correspondence: Meaghan O'Donnell, Australian Centre for Posttraumatic Mental Health, Level 3, Alan Gilbert Building 161 Barry Street, Carlton, VIC 3053 Australia. Email: mod@unimelb.edu.au

First received 11 Jul 2013, final revision 16 Jan 2014, accepted 14 Mar 2014

\section{References}

1 American Psychiatric Association. Diagnostic and Statistical Manual of Mental Disorders (5th edn) (DSM-5). APA, 2013.

2 Friedman MJ, Resick PA, Bryant RA, Strain J, Horowitz M, Spiegel D. Classification of trauma and stressor-related disorders in DSM-5. Depress. Anxiety 2011; 28: 737-49.

3 Brewin C. World Health Organization Preparation of ICD-11: clinical utility of diagnostic criteria for trauma related disorders, Part 1. Diagnosing PTSD from three core elements. Presented at the 28th Annual Meeting of the International Society for Traumatic Stress Studies, 1-3 November 2012, Los Angeles, USA (http://www.istss.org/Expert_Training_ISTSS_Conferences/ 5920.htm).

4 Maercker A, Brewin CR, Bryant RA, Cloitre M, Reed GM, van Ommeren M, et al. Proposals for mental disorders specifically associated with stress in the International Classification of Diseases-11. Lancet 2013; 381: 1683-5.

5 American Psychiatric Association. Diagnostic and Statistical Manual of Mental Disorders (4th edn, text revision) (DSM-IV-TR). APA, 2000.

6 World Health Organization. The ICD-10 Classification of Mental and Behavioural Disorders: Clinical Descriptions and Diagnostic Guidelines. WHO, 1992.

7 American Congress of Rehabilitation Medicine. Definition of mild traumatic brain injury. J Head Trauma Rehabil 1993; 8: 86-7.

8 Baker SP, O'Neil B, Haddon W, Long WB. The Injury Severity Score: a method for describing patients with multiple injuries and evaluating emergency care. J Trauma 1974; 14: 187-96.

9 Blake DD, Weathers FW, Nagy LM, Kaloupek DG, Charney DS, Keane TM. Clinician-Administered PTSD Scale for DSM-IV. National Center For Posttraumatic Stress Disorder, 1998.
10 Gabbe B, Harrison J, Lyons R, Jolley D. Modelling long term disability following injury: comparison of three approaches for handling multiple injuries. PLOS One. 2011; 6: e25862.

11 Weathers FW, Keane TM, Davidson J. Clinician-administered PTSD scale: a review of the first ten years of research. Depress Anxiety 2001; 13: 132-56.

12 Aziz M, Kenford S. Comparability of telephone and face-to-face interviews in assessing patients with posttraumatic stress disorder. J Psychiatr Pract 2004; 10: $307-13$.

13 Sheehan DV, Lecrubier $Y$, Harnett-Sheehan $K$, Amorim $P$, Janavs J, Weiller $E$, et al. The Mini International Neuropsychiatric Interview (M.I.N.I.): the development and validation of a structured diagnostic psychiatric interview. J Clin Psychiatry 1998; 59 (suppl 20): 22-33.

14 Nagi S. An epidemiology of disability amoung adults in the United States. Milbank Mem Fund Q Health Soc 1976; 54: 439-68.

15 WHODAS Group. World Health Organization Disability Assessment Schedule II. WHO, 2000.

16 Perini SJ, Slade T, Andrews G. Generic effectiveness measures: sensitivity to symptom change in anxiety disorders. J Affect Disord 2006; 90: 123-30.

17 Andrews G, Kemp A, Sunderland M, Von Korff M, Ustun T. Normative data for the 12 item WHO disability assessment schedule 2.0. PLOS One 2009; 4: e8343.

18 WHOQOL Group. Development of the World Health Organisation WHOQOL-BREF Quality of Life Assessment. Psychol Med 1998; 28: 551-8.

19 Hawthorne G, Herrman H, Murphy B. Interpreting the WHOQOL-Brèf: preliminary population norms and effect sizes. Soc Indic Res 2006; 77: 37-59.

20 Forbes D, Fletcher S, Lockwood E, O'Donnell M, Creamer M, Bryant RA, et al. Requiring both avoidance and emotional numbing in DSM-V PTSD: will it help? J Affect Disord 2011; 130: 483-6.

21 Elhai J, Ford JD, Ruggiero KJ, Frueh CB. Diagnostic alterations for posttraumatic stress disorder: examining data from the National Comorbidity Survey Replication and National Survey of Adolescents. Psychol Med 2009; 39: 1957-66.

22 Aversa LH, Stoddard JA, Doran NM, Au S, Chow B, McFall M, et al. PTSD and depression as predictors of physical health-related quality of life in tobacco-dependent veterans. J Psychosom Res 2012; 73: $185-90$.

23 Brewin CR. The nature and significance of memory disturbance in posttraumatic stress disorder. Annu Rev Clin Psychol 2011; 7: 203-27.

24 Bryant RA, O'Donnell ML, Creamer M, McFarlane AC, Silove D. Posttraumatic intrusive symptoms across psychiatric disorders. J Psychiatr Res 2011; 45: $842-7$.

25 Bryant RA, O'Donnell ML, Creamer M, McFarlane AC, Clark CR, Silove D. The psychiatric sequelae of traumatic injury. Am J Psychiatry 2010; 167: 312-20.

26 O'Donnell ML, Creamer M, Pattison P. Posttraumatic stress disorder and depression following trauma: understanding comorbidity. Am J Psychiatry 2004; 161: 1390-6.

27 Galatzer-Levy IR, Bryant RA. 636,120 ways to have posttraumatic stress disorder: the relative merits of categorical and dimensional approaches to posttraumatic stress. Perspect Psychol Sci 2013; 8: 651-62.

28 Baldessarini RJ, Finklestein S, Arana GW. The predictive power of diagnostic tests and the effect of prevalence of illness. Arch Gen Psychiatry 1983; 40: 569-73. 